\title{
The 14th European Workshop on Drug Metabolism
}

\begin{tabular}{l|l|l} 
F.K. & Frank K. & Jugert
\end{tabular}

Frank K. Jugert, Experimental Dermatology, Department of Dermatology, Medical Faculty of RWTH Aachen, 52074 Aachen (Germany)

The 14th European Workshop on Drug Metabolism of the European Society for Biochemical Pharmacology was held in Paris from July 4th to 8th, at the Medical Faculty of the University Rene Descartes. The meeting, organized perfectly by Philippe Beaune, was attended by approximately 600 scientists, with a growing number of colleagues from Eastern Europe. Its main focus was on all enzymatic processes taking place in the metabolisms of exogenous and endogenous substances. Lectures gave a broad overview over: different human flavin-containing monooxygenase species (R. Gasser); the two isozymes of the mitochondrial monoamine oxidase, which are expressed in all human tissues (Ph. Urban), and the regulation of the cytosolic $\mathrm{NAD}(\mathrm{P}) \mathrm{H}$ :quinone reductase (NQR) gene expression, which is controlled by at least two regulatory elements (RE), the xenobiotic RE and the antioxidant RE (C. Pickett). As we know that the NQR is very active in the skin, the ability of stimuli such as planar aromatic compounds, hydrogen peroxide, phorbol esters and phenolic antioxidants to induce the NQR activity is increasing in importance. The multigene family of the UDP-glucoronosyl transferases, mainly located in the endoplasmatic reticulum of the liver, have been described by S. Fournel-Gigleux. M. Coughtrie introduced the three main forms of the human cytosolic sul-fotransferases and showed that the sulfur esters of aromatic hydroxylamines, hydroxyaminic acids and the benzylic alcohols of polyaromatic hydrocarbons are ultimately carcinogens or mutagens generated by this enzyme. Thus he recommended drinking red wine prior to eating charbroiled meat, because red wine has the capacity to inhibit the main hepatic form of the sulfotransferase. Another gene superfamily providing protection against cytotoxic and genotoxic electro-philes, the glutathione S-transferases (B. Ketterer), was extended by the discovery of a fourth gene family (the-ta). Liver cancer associated with aflatoxin $\mathrm{B}$ exposition and the polymorphism in both the mu and theta families suggest possibilities for clinical trials.

The keynote lecture given by F.P. Guengerich on Human Cytochromes P450 highlighted the extraordinary amount of more than 300 P450 cDNAs cloned until now. Although humans express more than 40 P450s, between 25 and 60\% of the P450s in the liver are represented by the isozyme 3A4. There is a 20 -fold variability within this enzyme and it has no polymorphism. A wide range of xenobiotics, e.g. macrolide antibiotics such as cyclosporin, steroids, glucocorticoids, aflatoxin, terfenadine and acetaminophen, are metabolized. The variability of drug metabolism was shown by lectures on: Pharmacogenetics (U.A. Meyer); Drug Resistance and Drug Metabolism (A. Gouyette); Drug Metabolism and Pathology (P. Morselli), and Epidemiological Studies in Drug Conjugation (GJ. Mulder). A. Rane introduced The Individuality and Drug Specificity in Developmental Patterns of Metabolic Pathways, with different enzymes as examples: P450 2E1 is not expressed in fetal, but in all adult human livers. The Inhibition Drug Metabolism and Drug Interactions were explained practically by A. Boobis, 
showing e.g. the irreversible inhibiting effect of furafyllin on P450 1A2 and its dramatic result. As caffeine is metabolized by this isozyme, it cumulates to toxic blood concentrations in a few days. C.R. Wolf showed the Relationships between Endogenous Factors Such As Cytokines and Drug Metabolism.

Although hormones and diet have been shown to act on the enzymes, the second keynote lecture, given by D. Mansuy on The Great Diversity of Reactions Catalyzed By Drug-Metabolizing Enzymes, set the basis for understanding the chemical action of drug-

metabolizing enzymes. The session on drug toxicity was introduced by F. Oesch with Genotoxicity; in his lecture on Immune-Mediated Drug Toxicity, G. Ken-na showed serum antibodies from patients with drug-allergic/toxic reactions, binding to proteins of drug-treated animals; H. Sies lectured on Singlet Molecular Oxygen and D. Pessayre on Clinical Aspects. Practical approaches were presented in the session Which Tool For Which Applications, with themes such as Enzymes Expressed in Microorganisms (D. Pompon), Metabolism-Competent Human Cell Lines for More Predictive Risk Assessment (A. Pfeifer), Organ Slices to Investigate Drug Biotransformation (A. Vickers) and Side-Effects and Perfused Organs (P. Moldeus). The session was continued by A. Guillouzos with Human Cells in Culture, which showed the complex problems generated by this promising tool: primary or cell line, low proliferating capacity and dedifferentiation, and the loss of functions, especially in hepatocytes. Some of these problems do not exist in the case of skin drug metabolism systems such as keratinocyte cultures and skin equivalents. A critical approach on In vitro-in vivo Extrapolations was made by C. Bäärnhielm. The session was completed by: Transgenic Animals for Mu-tagenesis Studies by J. Van Delft and In vivo Metabolism Studies by D. Breimer.

The third keynote lecture given by P. Maurel on the Induction of Drug-Metabolizing Enzymes showed the inducers and the mechanisms of actions which keep the P450 working as hard as the invited speakers. The last day was reserved exclusively for extrahepatic drug metabolism, and B. Testa gave examples for Drug Penetration and Metabolism in the Brain with several mathematical formulas to evaluate the structure-penetration relationships of drugs. P.B. Watkins provided evidence that intestinal drug metabolism is a first pass for many drugs, such as oral cyclosporin, which is metabolized by P450 3A4 in the outer cells of the intestinal villi. This isozyme is only present in mature cells and is inducible by rifampicin. Crypt cells do not contain 3A4, so carcinogenic adducts with aflatoxin made by P450 are present only in the top cell layer, demonstrating the barrier function of the enzyme. The lecture on Drug Metabolism in the Skin was given by H. Merk. Metabolizing enzymes are a prerequisite for metabolism; examples for the expression and the catalytic activity of these enzymes in the skin were given, with special emphasis on drug metabolism. He also pointed out new developments regarding cell culture techniques with human keratinocytes and skin equivalents used in dermatopharmacology and dermatotoxicology and studies on the relationship between cytochrome P450 expression and cell differentiation processes. The

last session on the Implications of Drug Metabolites for Clinical and Drug Devleopment returned to practical aspects.

Five practical/educational sessions - Molecular Biology Applied to Drug Metabolism; Analytical Tools for Drug Metabolism; Which Isoenzymes Produce Which Metabolites; Covalent Binding, Toxicology and Immunotoxicology; and Molecular Modeling and Drug Metabolism - presented the opportunity to become acquainted with some methods and aspects of drug metabolism studied in the laboratories. 
Last but not least, the attendees could choose between 266 poster presentations in two sessions. To mention just a few of the many highlights: Eickelmann et al. developed a PCR assay to show the lack of the NQR which is correlated with resistance towards mito-mycin C, pointing out that this null allele in a German population may have relevance for the predisposition to renal cell carcinoma. Carriere et al. reported that the three different allelic forms of the human P450 2E show no relationship with the protein expression or the activity. Bouters et al. was able to use the baculovirus for the cDNA-directed expression of human P450 3A4 in SF9 insect cells; as this technique is known to produce in vivo-like proteins, it seems to be an advantage over other expression systems. Kaminski et al. employed capillary electrophoresis with argon-laser-induced fluorescent detection to quantify RT-PCR products down to attomoles product/ $\mu \mathrm{g}$ RNA concentrations.

This extraordinary meeting resulted in a widened knowledge in the interesting field of drug metabolism; we look forward to meeting some of the readers oíSkin Pharmacology in Jena, Germany, for the 15th European Workshop on Drug Metabolism in 1996. 